\title{
Uma análise comportamentalista de relatos verbais e práticas educativas parentais: alcance e limites
}

\author{
Vanessa Barbosa Romera Leme \\ Alessandra Turini Bolsoni-Silva ${ }^{1}$ \\ Kester Carrara \\ Universidade Estadual Paulista Júlio de Mesquita Filho, Bauru-SP, Brasil
}

\begin{abstract}
Resumo: O presente artigo discute a relevância de algumas estratégias metodológicas da Análise Aplicada do Comportamento para examinar práticas educativas parentais. Um instrumento e um conceito relacionados à coleta e análise de dados são discutidos sob a ótica teórica e aplicada: relatos verbais e análise funcional comportamental descritiva. Conclui-se que, consideradas as limitações dos relatos, as informações obtidas com essa estratégia e com a análise funcional comportamental descritiva permitem inferir algumas hipóteses sobre os comportamentos de pais e de filhos que podem ser utilizadas em ações que procuram investigar e intervir sobre os comportamentos problema e as habilidades sociais.
\end{abstract}

Palavras-chave: relações pais-criança, análise do comportamento, comunicação verbal.

\section{A behavioral analysis of verbal reports and parental educative practices: scope and limits}

\begin{abstract}
This study addresses the relevance of some methodological strategies of the Applied Behavior Analysis to examine parental educative practices. An instrument and a concept related to the data collection and analysis are discussed from a theoretical and applied perspective: verbal reports and descriptive functional behavioral analysis. The conclusion, considering the limitations of the reports, is that the information acquired using this strategy in addition to the descriptive functional behavioral analysis permitted the inference of some hypotheses concerning the behavior of parents and children, which can be used in actions aimed to investigate and intervene on behavioral problems and social skills.
\end{abstract}

Keywords: parent child relations, behavioral assessment, verbal communication.

\section{Un análisis conductista de los informes verbales y las prácticas educativas de los padres: alcances y límites}

\begin{abstract}
Resumen: El estudio analiza la importancia de algunas estrategias metodológicas del Análisis Aplicado de la Conducta en el examen de prácticas educativas de los padres. Un instrumento y un concepto relacionados con recopilación y análisis de los datos se examinan desde la óptica teórica y aplicada: informes verbales y análisis funcional de la conducta descriptiva. Concluimos que, teniendo en cuenta las limitaciones de los relatos, el resultado obtenido con esta estrategia y el análisis funcional de la conducta descriptiva permiten algunas hipótesis sobre la conducta de los padres y los niños que pueden ser utilizados en las acciones que tratan de investigar e intervenir sobre los problemas de comportamiento y las habilidades sociales.
\end{abstract}

Palabras clave: relaciones padres-niños, análisis de la conducta, comunicación verbal.

O analista do comportamento, ao realizar investigações e intervenções, especialmente fora do laboratório, defrontase com a necessidade de buscar informações sobre as condições de interação de seu cliente com o contexto ambiental onde vive. Nesse percurso, muitas vezes não pode prescindir da utilização de relatos verbais, uma vez que constituem fonte relevante de informações que ajudam a descrever o tipo e a forma de contingência vigente em cada interação estudada.

Ao analisar algumas pesquisas (Barry, Frick, \& Grafeman, 2008; Cia, Pamplin, \& Del Prette, 2006; Kerr, Lopez, Olson, \& Samerro, 2004; Oliveira, Rabuske, \& Arpini, 2007; Prinzie, Onghena, \& Hellinckx, 2007; Snyder, Cramer, Afrank, \& Patterson, 2005) que, dentre seus objetivos, procuram

\footnotetext{
1 Endereço para correspondência:

Profa. Dra. Alessandra Turini Bolsoni-Silva. Universidade Estadual Paulista. Faculdade de Ciências. Departamento de Psicologia. Av. Eng. Luiz Edmundo Carrijo Coube, 14-01. CEP 17.033-360. Bauru-SP, Brasil.

E-mail: bolsoni@fc.unesp.br
}

investigar as práticas educativas parentais, observou-se que o método empregado na coleta de dados caracterizava-se pelo uso de questionários, entrevistas e escalas, instrumentos que tomam como dado básico o relato verbal. Tal método é, por vezes, utilizado na Análise Aplicada do Comportamento, uma forma de atuação que pode contribuir com a investigação das interações entre pais e filhos, identificando contingências relacionadas às práticas educativas parentais. Nesse contexto, o presente artigo pretende discutir a relevância de algumas estratégias metodológicas que são utilizadas pela Análise Aplicada do Comportamento para examinar as práticas educativas parentais, enfatizando, de modo particular, determinadas implicações dos procedimentos de coleta e interpretação de dados provenientes dos relatos verbais.

\section{Análise funcional e relatos verbais}

O Behaviorismo Radical é definido por Skinner (1974/2004) como a filosofia de sua ciência, a Análise do 
Comportamento. Examinando a conceituação de Skinner, Abib (2001) afirma que o Behaviorismo seria uma filosofia do comportamento humano que apresenta um discurso sobre ciência. Nesse sentido, seria capaz de discutir e verificar questões epistemológicas a respeito de conceitos complexos como cultura, linguagem, pensamento, política e ética. Portanto, o Behaviorismo contempla um conjunto de pressupostos para além do campo empírico, que é condição necessária para o avanço científico da Análise do Comportamento, abrangendo esta, segundo Carrara (2008), uma área, um programa de pesquisa e uma atuação prática.

Carrara (2008) atribui três especificidades de atuação à Análise do Comportamento, a saber: (a) Análise Experimental do Comportamento que prioriza na dimensão da pesquisa, o delineamento de sujeito único e condições que maximizam o controle de variáveis; (b) Análise Conceitual do Comportamento que contempla investigações teóricas, filosóficas e epistemológico-metodológicas; (c) Análise Aplicada do Comportamento que seria o campo de intervenção planejada dos analistas do comportamento.

Independentemente da área de atuação, seja no laboratório, seja na clínica, os pressupostos lógicos do Behaviorismo são os mesmos, isto é, prevalece a lógica da seleção pelas consequências, ao mesmo tempo em que são mantidos os princípios básicos de extinção, reforçamento, punição, controle de estímulos, dentre outros. Assim, a Análise do Comportamento é constituída por um conjunto de princípios descritivo-explicativos derivados de experimentos de laboratório e de campo que pretende descrever, principalmente, as condições sob as quais os comportamentos são instalados, como se mantêm ou como se alteram (Carrara, 2008). Para realizar essa tarefa, a análise funcional é um recurso instrumental importante na compreensão das interações entre o organismo e o ambiente, por meio do comportamento.

Ao investigar os trabalhos de Skinner, alguns pesquisadores (Andery, Micheletto, \& Sério, 2001; Meyer, 1997; Vandenberghe, 2004) afirmam que o conceito de análise funcional passou por variações ao longo do tempo, indicando a necessidade de que estudos conceituais sejam realizados (Sturmey, 1996). Andery e cols. (2001) ao estudarem o conceito de análise funcional na obra de Skinner destacaram, por exemplo, que de 1931 a 1947, esse autor usou a expressão relação funcional, num sentido que incluiria tanto correlações obtidas por tratamentos estatísticos sem controle experimental de variáveis quanto descrições das relações entre eventos obtidas através da manipulação experimental. Nas palavras das autoras: "a expressão relação funcional parece se referir à atividade de descrever o fenômeno em estudo, e descrever envolve o estabelecimento, a descoberta de relações, não importando como estas relações foram identificadas" (p. 153, grifo das autoras). Contudo, em 1953, em Ciência e comportamento humano, segundo as autoras, Skinner usou o termo análise funcional restringindo-o à manipulação experimental de variáveis para demonstrar relações funcionais, isto é, dependência e regularidade entre as variáveis independentes e dependentes, em contrapartida a relações causais.
Andery e cols. (2001) informam que Skinner (1978/1957) surpreendeu com o livro Comportamento verbal, no qual propôs a possibilidade de uma análise funcional do comportamento verbal. As autoras destacaram que Skinner, nessa publicação, procedeu a uma discussão epistemológica que sugeria que o primeiro passo para a realização da análise funcional do comportamento verbal fosse sua descrição (topografia) e, num segundo momento, seria necessário realizar a sua explicação, isto é, explicitar quais seriam as variáveis das quais o comportamento verbal é função. Adicionalmente, em Ciência e comportamento humano, Skinner usou alguns conceitos obtidos com as pesquisas da Análise Experimental do Comportamento para discutir cultura, comportamento social e agências controladoras, tais como, religião, família e educação.

Os questionamentos de Skinner sobre conceitos complexos, como o de cultura, são sistematizados teoricamente quando o autor propõe o modelo de seleção por consequências (Skinner, 1981). Nesse texto, Skinner propõe um princípio explicativo para o comportamento operante, rejeita a visão mecanicista de causalidade e vincula a análise funcional a uma noção selecionista. Com efeito, a análise funcional parte do pressuposto de que o comportamento humano é complexo e multideterminado, participando, concomitantemente, a ação de três instâncias: a filogênese, a ontogênese e a cultura.

Os estudos que buscam investigar o conceito de análise funcional tratam, em geral, de duas possíveis formas de interpretação do conceito. Na primeira, verifica-se que há autores (Hanley, Iwata, \& McCord, 2003; Haynes \& O’Brien, 1990; Matos, 1999) que afirmam que o analista do comportamento não deve buscar explicações de causa-efeito, mas descrever as relações entre o comportamento e o ambiente, enfatizando a dependência entre eles por leis que expressem sequências regulares de eventos; portanto, para esses autores as análises funcionais são idiográficas. Em outras palavras, procuram compreender as relações de dependência entre ambiente e comportamento de um único indivíduo. Tal posição assim interpreta a idéia de Skinner ao afirmar que o homem existe em sua experiência particular, e, dessa maneira, análises funcionais são complexas e, na interpretação de alguns autores, não podem ser generalizáveis a outros indivíduos, descartando-se investigações nomotéticas (Vandenberghe, 2004). Geralmente, a análise funcional vista dessa forma envolve a manipulação de variáveis em um contexto controlado, simplificado e artificial, como é o caso do laboratório (Hanley e cols., 2003).

Por outro lado, surgiram, a partir dos anos 1950, alguns estudos (Goldiamond, 1974/2002; Kanfer \& Saslow, 1976; Marçal, 2005; Mace, 1994; Meyer, 1997; Sturmey, 1996; Tourinho, 1995) com a finalidade de realizar uma investigação conceitual a respeito da análise funcional e implementá-la fora do laboratório, isto é, na clínica. Consequentemente, o interesse da prática da análise funcional no ambiente clínico evidenciou as peculiaridades do trabalho do analista do comportamento nesse contexto. Sturmey (1996) 
é um dos autores que estudou as implicações práticas do uso da análise funcional e propôs uma distinção entre a análise funcional comportamental experimental e a análise funcional comportamental descritiva.

Segundo Sturmey (1996), a primeira forma de análise caracteriza-se pela manipulação experimental de variáveis para demonstrar dependência e regularidade entre as variáveis independentes e a variável dependente. Já na análise funcional comportamental descritiva, não há manipulação direta das variáveis, mas sim a operacionalização dos comportamentos-alvo que devem ser descritos e agrupados em classes de respostas (mesma função); consequentemente, deve-se também fazer uma análise das contingências (de reforçamento e/ou punição) que mantêm os comportamentos. Esse tipo de análise, mais adequado a um contexto clínico, não possibilitaria testar as hipóteses, uma vez que apresenta uma natureza correlacional, contudo, permitiria avaliar um extenso número de comportamentos que posteriormente podem ser testados experimentalmente (Sturmey, 1996).

Não obstante, as intervenções realizadas fora do laboratório merecem algumas considerações, com especial destaque ao comportamento verbal. Para Meyer (1997), na clínica ou em outros ambientes que não permitem o controle direto e sistemático das variáveis, o analista depara-se com dificuldades adicionais para a identificação das unidades de análise, a definição de eventos antecedentes e consequentes e, também, com a falta de informações para a definição dessas classes de respostas. Muitas vezes, nesses contextos, o analista não pode observar diretamente os comportamentos que constituem o foco da intervenção, tendo, portanto, acesso apenas aos relatos verbais (Moroz e cols., 2005; Simonassi, Tourinho, \& Silva, 2001, Tourinho, 1995).

O comportamento verbal é um comportamento, como tantos outros, que muda o ambiente e é suscetível às consequências da alteração que provoca nele. Contudo, é um comportamento cujo controle pelas consequências é mediado pela intervenção de outra pessoa (Baum, 1994/1999). Na situação típica, os comportamentos do ouvinte e do falante envolvem eventos antecedentes, comportamento verbal e eventos consequentes que, integrados, constituem o "episódio verbal" (Skinner 1978/1957). A diferença do comportamento verbal em relação aos outros comportamentos operantes é que as consequências do primeiro são reguladas por práticas culturais. Estas, por sua vez, são compreendidas por Skinner (1970/1953), como "um conjunto de contingências de reforço mantido por um grupo, possivelmente formuladas por meio de regras ou leis" (p. 175). Em outras palavras, o ouvinte apresenta um comportamento que funciona como consequência para o comportamento do falante; tal comportamento consequenciador já foi instalado pela comunidade verbal. Apesar dessas peculiaridades, para Skinner (1978/1957), o comportamento verbal é da mesma natureza físico-funcional que qualquer outro comportamento (aberto ou encoberto) e, sendo assim, é resultado dos três níveis de seleção, isto é, da história da espécie, da história do indivíduo e da história da cultura (Skinner, 1981). Portanto, o relato verbal é passível de ser analisado funcionalmente na medida em que é considerado como dado que se apresenta como meio útil para interpretação e investigação dos fenômenos comportamentais (Neno, 2003).

Nessa direção, ao fazer uma análise distinta da apresentada por Andery, Micheletto e Sério (2001) a respeito do conceito de análise funcional, Abib (2001) conclui que em suas obras mais recentes, a partir de Comportamento verbal, Skinner ampliou o alcance dessa formulação, propondo a investigação de diversas áreas aplicadas que transcendiam o contexto do laboratório como, por exemplo, a educação, a clínica, a política e a religião, dando margem para uma investigação interpretativa a respeito do comportamento humano. Contudo, com referência a esses contextos, Skinner não anunciou claramente metodologias específicas de análise.

Pesquisadores que trabalham com a Análise Aplicada do Comportamento concordam que o contexto social aduz complexidade à descrição e à análise do comportamento verbal, bem como dos comportamentos privados e sociais de forma geral (Meyer, 1997; Moroz e cols., 2005; Simonassi e cols., 2001, Tourinho, 1995). Na interpretação de tais autores, para Skinner (1945), ainda que os eventos públicos e privados tenham a mesma natureza (fenômenos físicos), não há acesso direto aos últimos senão avaliando o que as pessoas dizem que pensam, sentem e fazem. Contudo, esses relatos podem ser imprecisos e, por vezes, incoerentes em relação ao que realmente ocorre em seus cotidianos.

Outra dificuldade encontrada no estudo do comportamento verbal refere-se ao fato de que ele é, por vezes, controlado por estímulos discriminativos que se encontram "no mundo privado, dentro da pele" (Passos, 2003). Discussões na Análise do Comportamento a respeito dos limites de acesso aos eventos privados, bem como sua correspondência com os comportamentos públicos, são encontradas em pesquisas nacionais e internacionais, como por exemplo, Paniagua (2004) e Tourinho (2007). Além disso, há autores de outras abordagens teóricas que apresentam diferentes explicações daquelas do Behaviorismo Radical para as dificuldades encontradas no uso do relato verbal. Dentre eles, pode-se citar Nisbett e Wilson (1977) que, ao trabalharem com a teoria de atribuição, defendem a tese de que há processos mentais subjacentes a todo comportamento, sendo que nem sempre os indivíduos têm acesso a eles e, portanto, não são capazes de relatá-los. Já os estudos de Engelmann (1998, 2002), na Psicologia Gestáltica, indicam que o relato verbal representa apenas uma parte da consciência e, dessa maneira, pode ser falso por não conseguir traduzir numa linguagem diferente percepções não-auditivas.

Todavia, ainda que instrumentos como questionários e entrevistas, que investigam, através dos relatos, as ações, os pensamentos e os sentimentos das pessoas, tenham suas limitações na descrição das contingências reais, alguns autores (Carrara, 2008) entendem que não devem ser sumariamente descartados, uma vez que esses instrumentos podem ser utilizados em conjunto com outras formas de coleta de dados, principalmente em associação com o procedimento 
de observação do comportamento, que constitui evidente prioridade metodológica do Behaviorismo Radical. O uso de tal estratégia de pesquisa, associando instrumentos que coletam dados a partir do relato verbal e técnicas de observação, parece ampliar a confiabilidade dos registros. O relato verbal também permite colher dados sobre a história de aprendizagem do indivíduo e de múltiplos comportamentos, permitindo levantar hipóteses que consideram, mais amplamente, os níveis de seleção propostos por Skinner (1981). Além disso, as limitações dos relatos não impedem que vários autores da Análise do Comportamento (Goldiamond, 1974/2002; Kanfer \& Saslow, 1976; Meyer, 1997; Sturmey, 1996) utilizem o relato em suas investigações, o que respalda o uso desse tipo de recurso na coleta de dados.

Complementarmente, os instrumentos que se baseiam em relatos, isto é, no comportamento verbal, são importantes, pois: (a) auxiliam na descrição de contingências que fazem parte da história do indivíduo, ajudando a entender o comportamento presente; (b) permitem trabalhar com um conjunto extenso de variáveis de uma única vez, que podem ajudar na delimitação de comportamentos futuros que poderão, então, ser observados e manipulados com mais amplo controle metodológico (Carrara, 2008). Portanto, as pesquisas que transitam na Análise Aplicada do Comportamento podem se valer de vários instrumentos (ecletismo instrumental que não pode ser confundido com ecletismo teórico-epistemológico), os quais contribuem com fontes importantes de informações sobre os comportamentos sem, contudo, desviar dos pressupostos teóricos e metodológicos do Behaviorismo Radical (Carrara, 2008).

Conforme sinalizado anteriormente, as especificidades do trabalho realizado na Análise Aplicada do Comportamento fizeram surgir vários modelos de avaliação e de aplicação da análise funcional, uma vez que os terapeutas comportamentais buscam coletar dados, muitas vezes, por meio do relato verbal (entrevistas, escalas e questionários).

Kanfer e Saslow (1976) elaboraram um roteiro de entrevista para realizar um diagnóstico comportamental que considerasse, num primeiro momento, a análise da situaçãoproblema. Segundo os autores, as informações coletadas devem ser organizadas nas categorias de déficits, excessos e reservas comportamentais. Déficits comportamentais podem ser definidos como uma classe de respostas que deixa de ocorrer com suficiente frequência, com intensidade adequada e da maneira apropriada ou sob condições socialmente previstas. Já os excessos comportamentais são considerados enquanto classe de respostas que ocorre em excesso na frequência, intensidade e duração, ou sob condições em que a frequência aceita é próxima de zero. Por fim, as reservas comportamentais são uma classe de respostas não problemáticas, ou seja, são respostas socialmente esperadas que podem ser usadas para o desenvolvimento de novos repertórios no indivíduo. Em seguida, são averiguadas para cada uma das variáveis as situações em que elas ocorrem, as consequências e as possíveis mudanças que ocorreriam na vida do indivíduo, caso a terapia tivesse ou não sucesso. Juntamente com essas informações, os autores procuram investigar, além dos comportamentos-problema, a motivação do cliente, o comportamento de autocontrole, os relacionamentos sociais e o ambiente sócio-físico-cultural.

Com propósito semelhante, Goldiamond (1974/2002) propõe o Modelo Construcional, ao criticar, na década de 1960, as intervenções de modificação de comportamento. Conforme o autor, esse modelo procura não utilizar procedimentos que eliminem padrões comportamentais, mas que se desenvolvam vários comportamentos alternativos, usando, para identificar esse repertório, o instrumento que denominou de Questionário Construcional. De forma geral, o Modelo Construcional, também baseado nos princípios da Análise do Comportamento, focaliza a construção e ampliação de novos repertórios socialmente adequados, tais como, as habilidades sociais, por meio de procedimento de reforçamento positivo, da modelagem por aproximações sucessivas e do auto-registro de comportamentos.

A título de exemplo, uma criança pode obter atenção fazendo birras (problema de comportamento) ou solicitando, aos pais, que brinquem com ela (habilidade social) ou então pode agredir um colega quando quer algo (problema de comportamento) ou pode pedir que lhe empreste o que deseja (habilidade social). Portanto, quando os pais e os filhos aprendem outras formas de obter reforçadores através de comportamentos socialmente habilidosos, os comportamentos problema e as práticas educativas negativas podem perder sua funcionalidade, o que faria com que fosse reduzida sua ocorrência. Note-se que, neste estudo, comportamentos socialmente habilidosos e habilidades sociais são utilizados como sinônimos. As habilidades sociais são definidas por Del Prette e Del Prette (2005) como "diferentes classes de comportamentos sociais do repertório de um indivíduo, que contribuem para a competência social, favorecendo um relacionamento saudável e produtivo com as demais pessoas" (p. 31). Os mesmos autores sinalizam um conjunto de habilidades sociais na infância, tais como autocontrole, expressividade emocional, empatia e fazer amizades, entre outras.

\section{Análise Aplicada do Comportamento e práticas educativas parentais}

As propostas de Kanfer e Saslow (1976) e Goldiamond (1974/2002) trabalham com um conjunto complexo de informações que não pode ser investigado através de um modelo de análise funcional realizado num contexto de laboratório, pois o pesquisador não consegue manipular as variáveis da mesma maneira, já que não tem acesso direto às experiências vividas pelo indivíduo, tendo contato apenas com o relato (De Rose, 1999). Ao mesmo tempo, os autores evidenciam a importância de avaliar não somente os comportamentos problema, mas também os socialmente habilidosos, pois se busca uma descrição de interdependências comportamentais, na tentativa de avaliar mais amplamente o repertório do indivíduo. 
Assim, considerando que os comportamentos de pais e filhos são multideterminados por fatores tais como condições sócioeconômicas, estrutura familiar, relacionamento conjugal, gênero parental e temperamento infantil (Webster-Stratton, Reid, \& Hammond, 2004), acredita-se que as propostas de Kanfer e Saslow (1976) e Goldiamond (1974/2002), juntamente com a análise funcional comportamental descritiva (Sturmey, 1996), podem ser utilizadas para se estudar as práticas educativas parentais, a partir da Análise Aplicada do Comportamento.

Vários são os termos encontrados na literatura nacional (Gomide, 2003) e internacional (Baumrind, 1971; Darling \& Steinberg, 1993) para identificar e classificar as estratégias educativas que os pais utilizam no processo de socialização dos filhos. Segundo alguns autores (Darling \& Steinberg, 1993; Pacheco, Silveira, \& Schneider, 2008) tais terminologias não são incompatíveis entre si, especialmente quando se considera que todas, de forma geral, procuram investigar aspectos da interação entre pais e filhos. Além disso, conforme Pacheco e cols. (2008), há autores que utilizam, por exemplo, as expressões estilos parentais e práticas educativas parentais como sinônimos (Gomide, 2003) e outros não (Baumrind, 1971; Darling \& Steinberg, 1993).

As práticas educativas parentais constituem, para Gomide (2003), um conjunto de estratégias e técnicas utilizadas pelos pais para orientar os comportamentos dos filhos. A mesma autora afirma que os estilos parentais podem ser compreendidos como um conjunto de práticas educativas, as quais podem ser divididas em dois grandes grupos: o primeiro corresponde às práticas que promovem o desenvolvimento de comportamentos socialmente habilidosos (práticas educativas positivas, tais como uso adequado de atenção, de distribuição de privilégios, do estabelecimento de regras, dentre outras), o segundo grupo refere-se às que podem favorecer o surgimento de problemas de comportamento (práticas educativas negativas, que implicam, por exemplo, ausência de atenção e afeto, práticas corporais negativas, uso de ameaça ou chantagem de abandono, dentre outras). Portanto, podese concluir que, consideradas suas peculiaridades, os estilos parentais e as práticas educativas parentais referem-se, de forma, geral, a determinados comportamentos que os pais utilizam para educar os filhos, que podem, conforme Gomide (2003), tanto contribuir com o surgimento e com a manutenção dos problemas de comportamento, quanto com o desenvolvimento das habilidades sociais dos filhos.

Pesquisas (Kerr e cols., 2004; Schoppe, Mangelsdorf, \& Frosch, 2001; Vasconcelos \& Souza, 2006) indicam que os pais apresentam dificuldades em estabelecer limites para os filhos, valendo-se de práticas educativas negativas (tais como agressões físicas ou verbais e ameaças de punição) que podem gerar diversas consequências deletérias às relações entre pais e filhos e ao desenvolvimento destes, como o surgimento de problemas de comportamento. Não há um consenso na literatura quanto à definição, à classificação e ao diagnóstico para os problemas de comportamento, contudo, neste trabalho, esses serão entendidos como: déficits e/ou excessos comportamentais que prejudicam a interação da criança com pares e adultos (...) e que dificultam o acesso da criança a novas contingências de reforçamento, que por sua vez, facilitariam a aquisição de repertórios relevantes de aprendizagem. (Bolsoni-Silva, 2003, p.10)

A gravidade dos prejuízos decorrentes do caráter usual das práticas educativas negativas nas relações pais-filhos é corroborada pelos resultados de estudos (Campezatto \& Nunes, 2007; Melo \& Perfeito, 2006) que indicam que a procura por atendimento psicológico, seja através dos cuidadores da criança, seja por indicação da escola, em clínicas, é motivada principalmente por queixas de agressividade e violência.

Diante de tal demanda por atendimento psicológico, alguns pesquisadores (Oliveira e cols., 2007; Barry e cols., 2008; Prinzie e cols., 2007; Snyder e cols., 2005) têm procurado identificar quais comportamentos parentais poderiam prevenir problemas comportamentais em crianças e adolescentes, enquanto outros (Marchant, Young, \& West, 2004; Phaneuf \& McIntyre, 2007; Shaw, Dishion, Supplee, Gardner, \& Arnds, 2006; Turner \& Sanders, 2006) têm implementado programas para tentar sanar essas dificuldades.

De forma geral, os estudos mencionados que se baseiam na Análise do Comportamento utilizam muitas vezes como estratégia metodológica, em conjunto ou não com a observação (natural ou experimental), o relato verbal (entrevistas, questionários e escalas) para investigar as práticas educativas parentais. Por exemplo, Marchant e cols., (2004) procuraram, por meio de observações realizadas nas residências de quatro famílias, verificar quão efetivamente os pais podem, após uma intervenção, aprender e aplicar técnicas de reforçamento, elogios efetivos e instrutivos, ensino direto e técnica corretiva com crianças desobedientes. Snyder e cols. (2005) realizaram um estudo para investigar as relações entre disciplina ineficiente e agressividade parental com o desenvolvimento de problemas de comportamento em pré-escolares. Para coletar os dados, os autores entregaram questionários às mães e aos professores das crianças. Complementarmente, realizaram observação das crianças em casa e nos playground das escolas. Em um outro estudo, Barry e cols. (2008) procuraram comparar os relatos dos pais e dos seus filhos (idade entre 9 e 15 anos) com problemas de comportamento, a respeito das práticas educativas parentais. Para isso, os autores solicitaram que os participantes respondessem a questionários e a inventários por um período de três anos.

Adicionalmente, estudos de revisão (Lundahl, Risser, \& Lovejoy, 2006; Morawska \& Sanders, 2005) que tiveram por objetivo avaliar a efetividade de treinamento parental, referenciado especialmente em técnicas da Análise do Comportamento, indicam que a maioria dos programas de intervenção com pais realiza a coleta de dados através da combinação de observação e relato verbal (Patterson, Mockford, \& StewartBrown, 2005; Webster-Stratton e cols., 2004) ou só do relato (Boogs e cols., 2004; Muntz, Hutchings, Edwards, Housome, 
\& O'Ceilleachair, 2004). Assim, verifica-se o uso frequente do relato verbal, utilizado em conjunto ou não com a observação, como procedimento para coleta de dados, o que pode facilitar a obtenção de informações sobre os comportamentos dos participantes (Laville \& Dionne, 1999), além de contribuir para a eficácia dos programas de intervenção.

Deve-se considerar que práticas parentais são, sobretudo, práticas culturais, cultura esta que é compartilhada e transmitida por um grupo de geração a geração através do comportamento verbal (Baum, 1994/1999). Isso mostra a relevância do uso do relato verbal como fonte de informações para favorecer a descrição de características de determinada prática cultural, o que pode servir de base para interpretações acerca da função de determinados comportamentos, reiterando uma tendência na literatura internacional avaliada.

Nessa direção, chamam a atenção alguns estudos nacionais (Bolsoni-Silva, Bitondi, \& Marturano, 2008; Leme, 2008; Villa-Boas, 2007) que procuraram avaliar o contexto das práticas educativas dos pais, das habilidades sociais e dos problemas de comportamento dos filhos. Sucintamente, esses trabalhos propuseram-se a, dentre outros objetivos, investigar mediante a utilização do relato verbal: (a) o contexto atual em que os pais e os filhos apresentavam seus comportamentos, por exemplo, "Em quais situações você expressa sentimentos positivos ao seu filho?", "Em quais situações seu filho faz pedidos?" e "Em quais situações seu filho briga com outras crianças?"; (b) as consequências apresentadas pelos pais e pelos filhos, por exemplo, "O que você faz quando seu filho a elogia?" e "O que seu filho faz quando você expressa sentimentos positivos?".

Para o analista do comportamento, as informações obtidas com os estudos anteriormente mencionados são imprescindíveis, pois possibilitam descrever episódios comportamentais a partir de circunstâncias antecedentes e consequentes ao comportamento que devem ser articuladas entre si e com o contexto (Carrara, 2008). Com isso é possível descrever as contingências sob as quais os comportamentos ocorrem ou deixam de ocorrer e, assim, há possibilidade de se modificá-las a partir da alteração do ambiente que contextualiza as intervenções. Dessa maneira, uma possibilidade para investigar as práticas educativas dos pais seria coletar informações não só dos comportamentos problema destes e de seus filhos, mas também dos socialmente habilidosos, segundo o Modelo Construcional de Goldiamond (1974/2002). Num segundo momento, esses dados poderiam ser examinados por meio do conceito de análise funcional comportamental descritiva (Sturmey, 1996), que indica a formulação de hipóteses sobre as variáveis das quais o comportamento é função.

\section{Considerações finais}

Ainda que a Análise do Comportamento tenha como metodologia de pesquisa a experimentação através do delineamento com sujeito único, são inegáveis os avanços realizados por pesquisadores que têm desenvolvido outras formas de investigação do comportamento humano em contextos fora do laboratório (em particular, mediante comparações inter-sujeitos), sem, contudo, negar os princípios do Behaviorismo Radical, principalmente a concepção skinneriana de seleção pelas consequências.

As informações coletadas a partir de relatos e da análise funcional comportamental descritiva permitem inferir, com razoável confiabilidade, algumas hipóteses sobre os comportamentos dos pais e dos filhos que, acredita-se, podem apresentar-se condizentes com a Análise Aplicada do Comportamento. Assim, um ecletismo instrumental, tal como é utilizado em muitas pesquisas com pais, embora útil e plausível, não pode prescindir dos fundamentos teóricos e metodológicos do Behaviorismo Radical (Carrara, 2008).

Do mesmo modo, a utilização de diversos instrumentos, ainda que baseado no relato verbal, o qual apresenta conhecidas limitações (por exemplo, as inconsistências entre o fazer e o dizer, causado, às vezes, pela falta de acurácia na "observação" dos próprios comportamentos da pessoa) contribui com a descrição do contexto, possibilitando hipotetizar algumas relações entre o indivíduo e o ambiente, mediadas pelo comportamento. Torna-se, então, possível sugerir algumas explicações, ao menos em parte, para as frequências, por exemplo, dos comportamentos que compõem o repertório de habilidades sociais e de problemas de comportamento das crianças e das práticas educativas dos pais. Tais informações podem contribuir para intervenções realizadas com pais que apresentam queixas de problemas de comportamento de seus filhos.

Ressalte-se que, para o Behaviorismo Radical, o comportamento é sempre multideterminado, ou seja, são diversas as variáveis que influenciam os comportamentos nas relações entre indivíduo e ambiente. Desse modo, tanto a literatura referenciada em trabalhos teóricos quanto a referenciada em estudos empíricos, parecem assegurar a relevância da utilização da Análise Aplicada do Comportamento na investigação das práticas educativas parentais, considerando o alcance e os limites do uso do relato verbal como instrumento de coleta de dados. Nessa perspectiva, diante da complexidade que envolve as interações entre pais e filhos, embora insuficiente quando usado isoladamente, revelam-se, em contrapartida, bastante úteis os relatos verbais, uma vez que contribuem para ampliar a compreensão das variáveis que envolvem as práticas educativas parentais, quando cuidadosamente e criteriosamente coletados. Além disso, as hipóteses de trabalho inferidas a partir das análises funcionais comportamentais descritivas (Sturmey, 1996), devem ser ponderadas e complementadas com estudos futuros, que podem envolver outras variáveis e outros delineamentos metodológicos.

\section{Referências}

Abib, J. A. D. (2001). Behaviorismo radical como pragmatismo na epistemologia. In H. J. Guilhardi, M. B. B. P. Madi, P. P. Queiroz, \& M. C. Scoz (Orgs.), Sobre comportamento e cognição (pp.158-161). Santo André, SP: Arbytes. 
Andery, M. A. P. A., Micheletto, N., \& Sério, T. M. de A. P. (2001). Análise funcional na análise do comportamento. In H. J. Guilhardi, M. B. B. P. Madi, P. P. Queiroz, \& M. C. Scoz (Orgs.), Sobre comportamento e cognição (pp. 148-157). Santo André, SP: Arbytes.

Barry, C. T., Frick, P. J., \& Grafeman, S. J. (2008). Child versus parents reports of parenting practices: Implications for the conceptualization of child behavioral and emotional problems. Assessment, 15, 294-303.

Baum, W. M. (1999). Compreender o behaviorismo: Ciência, comportamento e cultura (M. T. A. Silva, M. A. Matos, \& G. Y. Tomanari, Trads.). Porto Alegre: Artmed. (Original publicado em 1994)

Baumrind, D. (1971). Current patterns of parental authority. Developmental Psychology Monograph, 4, 1-103.

Boggs, S. R., Eyberg, S. M., Edwards, D., Rayfield, A., Jacobs, J., Bagner, D., \& Hood, K. (2004). Outcomes of parentchild interaction therapy: A comparison of treatment completers and study dropouts one to three years later. Child \& Family Behavior Therapy, 26(4), 1-22.

Bolsoni-Silva, A. T. (2003). Habilidades sociais educativas, variáveis contextuais e problemas de comportamento: Comparando pais e mães de pré-escolares. Tese de doutorado não-publicada, Universidade de São Paulo, Ribeirão Preto, SP.

Bolsoni-Silva, A. T., Bitondi, F., \& Marturano, E. M. (2008). Intervenção em grupo para pais: A importância do diagnóstico comportamental individual. In M. R. Cavalcante (Org.), Análise do comportamento: Avaliação e intervenção (pp. 79-100). São Paulo: Roca.

Campezatto, P. V. M., \& Nunes, M. L. T. (2007). Atendimentos em clínicas-escola de psicologia da região metropolitana de Porto Alegre. Estudos de Psicologia (Campinas), 24, 363-374.

Carrara, K. (2008). Bases conceituais revisitadas, implicações éticas permanentes e estratégias recentes em análise aplicada do comportamento. In M. R. Cavalcante (Org.), Avaliação e intervenção em análise do comportamento: Aspectos de procedimentos (pp. 1-14). São Paulo: Roca.

Cia, F., Pamplin, R. C. De O., \& Del Prette, Z. A. P. (2006). Comunicação e participação pais-filhos: Correlação com habilidades sociais e problemas de comportamento dos filhos. Paidéia (Ribeirão Preto), 16, 395-408.

Darling, N., \& Steinberg, L. (1993). Parenting styles as context: An integrative model. Psychological Bulletin, 113, 487-496.

De Rose, J. C. (1999). O relato verbal segundo a perspectiva da análise do comportamento. In R. A. Banaco (Org.), Sobre comportamento e cognição: Aspectos teóricos, metodológicos e a formação em análise do comportamento e teoria cognitivista (pp. 148-163). São Paulo: Arbytes.

Del Prette, Z. A. P., \& Del Prette, A. (2005). Psicologia das habilidades sociais na infância: Teoria e prática. Petrópolis, RJ: Vozes.
Engelmann, A. (1998). Ciência natural e consciência [Versão eletrônica]. Psicologia: Reflexão e Crítica, 11, 273280. Recuperado em 20 agosto 2007, de http://www. scielo.br/scielo.php?pid=S0102-79721998000200007$\&$ script $=$ sci_arttext

Engelmann, A. (2002). Da conceituação de estado subjetivo até a proposição dos escalões de percepto. Psicologia: Reflexão e Crítica, 15, 393-405.

Goldiamond, I. (2002). Toward a constructional approach to social problems: Ethical and constitutional issues raised by applied behavioral analysis. Behavior and Social Issues, 11, 108-197. (Original publicado em 1974)

Gomide, P. I. C. (2003). Estilos parentais e comportamento anti-social. In A. Del Prette \& Z. A. P. Del Prette (Orgs.), Habilidades sociais, desenvolvimento e aprendizagem: Questões conceituais, avaliação e intervenção (pp. 2161). Campinas, SP: Alínea.

Hanley, G. P., Iwata, B. A., \& McCord, B. E. (2003). Functional analysis of problem behavior: A review. Journal of Applied Behavior Analysis, 36, 147-185.

Haynes, S. N., \& O’Brien, W. C. (1990). Functional analysis in behavior therapy. Clinical Psychology Review, 10, 649-688.

Kanfer, F. H., \& Saslow, G. (1976). An outline for behavioral diagnosis. In E. J. Mash \& L. G. Terdal (Eds.), Behavior therapy assessment. New York: Springer Publishing Company.

Kerr, D. C. R., Lopez, N. L., Olson, S. L., \& Sameroff A. J. (2004). Parental discipline and externalizing behavior problems in early childhood: The roles of moral regulation and child gender. Journal of Abnormal Child Psychology, 32, 369-383.

Laville, C., \& Dionne, J. (1999). A construção do saber: Manual de metodologia da pesquisa em ciências humanas. Porto Alegre: Artmed.

Leme, V. B. R. (2008). Habilidades sociais e problemas de comportamento de pré-escolares e sua relação com as habilidades sociais educativas parentais. Dissertação de mestrado não-publicada, Universidade Estadual Paulista Júlio de Mesquita Filho, Bauru, SP.

Lundahl, B., Risser, H. J., \& Lovejoy, M. C. (2006). A metaanalysis of parent training: Moderators and follow-up effects. Clinical Psychology Review, 26, 86-104.

Mace, C. (1994). The significance and future of functional analysis methodologies. Journal of Applied Behavior Analysis, 27, 211-214.

Marçal, J. V. de S. (2005). Estabelecendo objetivos na prática clínica: Quais caminhos seguir? Revista Brasileira de Terapia Comportamental e Cognitiva, 7, 231-246.

Marchant, M., Young, R., \& West, R. P. (2004). The effects of parental teaching on compliance behavior of children. Psychology in the Schools, 41, 337-350.

Matos, M. A. (1999). Análise funcional do comportamento. Estudos de Psicologia (Campinas), 16, 8-18. 
Melo, S. A., \& Perfeito, H. C. C. S. (2006). Características da população infantil atendida em triagem no período de 2000 a 2002 numa clínica-escola. Estudos de Psicologia, 23, 239-249.

Meyer, S. B. (1997). O conceito de análise funcional. In M. Delitti. (Org.), Sobre comportamento e cognição: A prática da análise do comportamento e da terapia cognitivo-comportamental (pp. 31-36). Santo André, SP: Arbytes Editora.

Morawska, A., \& Sanders, M. R. (2005). Self-administered behavioral family intervention for parents of toddlers: Part I - Efficacy. Journal of Consulting and Clinical Psychology, 74, 10-19.

Moroz, M., Rubano, D. R., Lopes, A. L., Maurutto, A. A., Lucci, M. A., Utida, H. H., \& Bocchi, K. B. (2005). Verbalizações e o estudo de eventos encobertos: Discussão metodológica. Revista Brasileira de Terapia Comportamental e Cognitiva, 7, 179-195.

Muntz, R., Hutchings, J., Edwards, R, Hounsome, B., \& O'Ceilleachair,A.(2004). Economic evaluation of treatments for children with severe behavioural problems. The Journal of Mental Health Policy and Economics, 7, 177-89.

Neno, S. (2003). Análise funcional: Definição e aplicação na terapia analítico-comportamental. Revista Brasileira de Terapia Comportamental, 5, 151-165.

Nisbett, R. E., \& Wilson, T. D. (1977). Telling more than we can know: Verbal reports on mental process. Psychological Review, 84, 231-259.

Oliveira, D. S. de., Rabuske, M. M., \& Arpini, D. M. (2007). Práticas de educação: Relato de mães usuárias de um serviço público de saúde. Psicologia em Estudo, 12, 351-361.

Pacheco, J. T. B., Silveira, L. M. De O., \& Schneider, A. M. De A. (2008). Estilos e práticas educativas parentais: Análise da relação desses construtos sob a perspectiva dos adolescentes. Psico, 39, 66-73.

Paniagua, F. A. (2004). Utility of verbal-nonverbal correspondence-training techniques in outpatient pediatric settings. Psychological-Reports, 94, 317-326.

Passos, M. de. L. R. da F. (2003). Análise funcional do comportamento verbal em verbal behavior (1957) de B. F. Skinner. Revista Brasileira de Terapia Comportamental e Cognitiva, 5, 195-213.

Patterson, J., Mockford, C., \& Stewart-Brown, S. (2005). Parents' perceptions of the value of the webster-stratton parenting programme: A qualitative study of a general practice based initiative. Child: Care, Health and Development, 31, 53-64.

Phaneuf, L., \& McIntyre, L. L. (2007). Effects of individualized video feedback combined with group parent training on inappropriate maternal behavior. Journal of Applied Behavior Analysis, 40, 737-741.

Prinzie, P., Onghena, P., \& Hellinckx, W. (2007). Reexamining the parenting scale: Reliability, factor structure, and concurrent validity of a Scale for Assessing the Discipline Practices of mothers and fathers of elementary-schoolaged children. European Journal of Psychological Assessment, 23, 24-31.
Simonassi, L. E., Tourinho, E. Z., \& Silva, A. V. S. (2001). Comportamento privado: Acessibilidade e relação com comportamento público. Psicologia: Reflexão e Crítica, 14, 133-142.

Schoppe, S. J., Mangelsdorf, S. C., \& Frosch, C. A. (2001). Coparenting, family process, and family structure: Implications for preschoolers' externalizing behavior problems. Journal of Family Psychology, 15, 526-545.

Shaw, D. S., Dishion, T. J., Supplee, L., Gardner, F., \& Arnds, K. (2006). Randomized trial of a family-centered approach to the prevention of early conduct problems: 2-year effects of the family check-up in early childhood. Journal of Consulting and Clinical Psychology, 74, 1-9.

Skinner, B. F. (1945). The operational analysis of psychological terms. Psychological Review, 52, 270-277.

Skinner, B. F. (1970). Ciência e comportamento humano (J. C. Todorov \& R. Azzi, Trads.). São Paulo: Martins Fontes (Original publicado em 1953)

Skinner, B. F. (1978). O comportamento verbal (M. P. Villalobos, Trad). São Paulo: Cultrix. (Original publicado em 1957)

Skinner, B. F. (1981). Selection by consequences. Science, 231, 501-504.

Skinner, B. F. (2004). Sobre o Behaviorismo (M. P. Villalobos, Trad). São Paulo: Cultrix. (Original publicado em 1974)

Snyder, J., Cramer, A., Afrank, J., \& Patterson, G. (2005). The contributions of ineffective discipline and parental hostile attributions of child misbehavior to the development of conduct problems at home and school. Developmental Psychology, 41, 30-41.

Sturmey, P. (1996). Functional analysis in clinical psychology. England: John Wiley e Sons.

Tourinho, E. Z. (1995). O autoconhecimento na psicologia comportamental de B. F. Skinner. Belém: EdUFPA.

Tourinho, E. Z. (2007). Conceitos científicos e eventos privados como resposta verbal. Interação em Psicologia, 11, 1-9.

Turner, K. M. T., \& Sanders, M. R. (2006). Dissemination of evidence-based parenting and family support strategies: Learning from the triple P-positive program system approach. Aggression and Violent Behavior, 11, 176-193.

Vandenberghe, L. (2004). Análise funcional. In M. Z. S. Brandão, F. C. S. Conte, F. S. Brandão, Y. K. Ingberman, V. L. M. Silva, \& S. M. Oliani (Orgs.), Sobre comportamento e cognição: Contingências e metacontingências (pp. 62-71). Santo André, SP: ESETec Editores Associados.

Vasconcelos, A. C., \& Souza, M. B. (2006). As noções de educação e disciplina em pais que agridem seus filhos. Psico, 37, 15-22.

Villas Boas, A. C. V. B. (2007). Comportamento infantil, separação conjugal e habilidades sociais educativas parentais. Dissertação de mestrado não-publicada, Universidade Estadual Paulista Júlio de Mesquita Filho, Bauru, SP. 
Webster-Stratton, C., Reid, M. J., \& Hammond, M. (2004).

Treating children with early-onset conduct problems: Intervention outcomes for parent, child, and teacher training. Journal of Clinical Child and Adolescent Psychology, 33, 105-124.

Vanessa Barbosa Romera Leme é doutoranda do Programa de Pós-graduação em Psicologia da Faculdade de Filosofia, Ciências e Letras de Ribeirão Preto da Universidade de São Paulo, bolsista FAPESP.

Alessandra Turini Bolsoni-Silva é Professora Doutora da Faculdade de Ciências da Universidade Estadual Paulista Júlio de Mesquita Filho, campus Bauru.

Kester Carrara é Professor Doutor da Faculdade de Ciências da Universidade Estadual Paulista Júlio de Mesquita Filho, campus Bauru.

Recebido: $14 / 05 / 2008$

$1^{a}$ revisão: $14 / 10 / 2008$

$2^{a}$ revisão: 22/01/2009

$3^{a}$ revisão: 27/02/2009

Aceite final: 19/03/2009 